\title{
Pedestrian Gender Prediction using Machine Learning
}

\author{
K Divya \\ Dept. of CSE \\ SNIST \\ Hyderabad, India
}

\author{
K Damodhar Rao \\ Associate Professor, Dept. of CSE \\ SNIST \\ Hyderabad, India
}

\author{
Dr. Prasanta Kumar Sahoo \\ Professor, Dept. of CSE \\ SNIST \\ Hyderabad, India
}

\begin{abstract}
Gender recognition of a person is an important and difficult field in image processing. Computerised gender acknowledgement is a inspiring field where researches are been taking place. This research work has been used in variety of real time applications .These include intelligent surveilliance ,interaction between humans and robots, behaviour analysis of people, sum up number of males and females in a public place.Previous research works were not up to the mark as they have less accurracies in identifying the gender of a pedestrian.In this paper we have proposed a gender identification model through body appearance.The training and testing is performed on MIT dataset that contains both front and back views of a pedestrian.For classification purpose we used random forest algorithm on MIT dataset and this approach has achieved an accuracy of 97.5 percentage.
\end{abstract}

Keywords:Pedestrian, Machine learning, Threshold, Random forest algorithm, Gender Identification.

\section{I.INTRODUCTION}

A person or an individual who walks on the streets or may be walking or running can be said as pedestrian.Now a days it is a tough field in image processing for identifying the gender of a pedestrian and also there have been many analysis arising .Here for recognising the pedestrian gender we extract few features which are useful in his case they are like voice,clothes,body shapes,jewellery,skin colour and hair styles.Face identification requires only face for gender prediction but it fails for the pedestrian case as pedestrian pictures are received from the camera that are very distant from the pedestrians. This appears to have a low quality images of person overall. This has a reason where the pictures have appearance of low quality. It is said if the camera is backside of a person the method of face recognition system will fail.In certain situations we consider the hint for predicting the gender of a person on foot are whole body and appearance of a walker. In current events, the term shows someone walking around a road.

Walkers are the ones who have few extraordinary necessities to ensure their prosperity in transportation structure when they are travelling with others in few situations. As this is one of a special substantial in third world setting, it is fundamental to build a predominant comprehension of individual by walking rehearses in making countries.It can be said as the male delay time is half of the female delay time, and folks are twice as at risk to cause conflicts with motor vehicles. These type of strong models will in a perfect world assistance in the perception of individual by walking conduct in association with motor vehicle traffic in urban districts of making countries, encouraging increasingly secure crossing points to be organized. Arranging a person sexual direction is said to be extended thought in $\mathrm{PC}$ vision research starting late.

There are many different potential applications, for instance, in PC-human association, surveillance, and counting the walkers. Not only there has a great deal of works on seeing sexual orientation direction from facial information alone,But less work has been done on using signals from the whole body of a person. Only the face may not be attainable for security reasons in certain type of explicit conditions or as a result of deficient goals. Another simpler clarification can be as , the face is not observable from the back point of view of a person. Most facial gender identification systems rely upon constrained situations, frontal or close frontal point of views on the head are few examples. Consequently, we acknowledge that there are certain advantages on using the whole body.

Allow us to consider how we may have the alternative to perceive the gender direction of a walker subordinate in general human body as opposed to relying upon the face alone. As there are a lot of differentiations in the life structures between the men and women, the strong sign is said as body shape . Regardless, attire may cause snag, for instance, baggy pieces of clothing that make the body shape progressively timid. There are different type of clothing for different genders but anyways practically identical sorts are also worn by both, long pants or T-shirts as a example. Haircut that acts a strong sign in a large portion of instances, anyway length of the hair can be a sign of confusion. Notwithstanding this, individuals sexual direction can be isolated exactly. The robotized person on foot recognition system that identifies a pedestrian as a male or female is utilized in numerous sort of utilizations. For such conditions, we recognise the walker gender based upon the appearance .Few ongoing applications are given below:

1. Collaboration between people and robots,in such cases the robots need information regarding the gender to make legitimate correspondence with the individuals.

\section{Examining the actions or conduct of people in a mall.}

3.Verifying the person on foot wellbeing through smart transportation framework. 


\section{RELATED WORKS}

There are various classifications of gender identification techniques discovered. They are human face based step based, feelings based and human body based strategies. A large portion of the work is done on perceiving sex. These are acquired from face pictures. A not very many research articles group sexual orientation through human body appearance. As the pictures are taken from a separation the video reconnaissance cameras, in most part of cases have low goals. Faces are exceptionally hard to break down as in the case of low goals pictures. There are few human body traits like dress, body, walk and shape, and these are useful and are valuable even in low goals situations.

There are factors that influence sex characterization closeness of body shape, dress styles and haircuts of the two guys and females. Notwithstanding these components, few difficulties are present varieties, impediment in pictures, varieties in age, diminish goals of far off pictures and picture noising.

For performing the sex based characterization this method has square chart of the proposed approach. In the going with area, detail conversation of all means of proposed system is introduced.

Sun Yanhui and Bo Chunjuan et al. proposed a novel face picture gender characterization calculation is relied on svm. As of removing the RPPBTF, the picture premise capacities have been received that is acquired by Principal Component Analysis. Lot of layouts for getting surface examples. Contrasted with the conventional PPBTF technique, the RPPBTF calculation utilizes a delicate administrator as opposed to a hard administrator for extricating surface highlights. This change makes that the RPPBTF strategy not just keeps up the upside of the first PPBTF technique yet in addition removes a lot of surface data absent a lot of computational burden. Exploratory outcomes and dialogs show that the proposed strategy is superior to anything other prevalent highlights for the gender orientation characterization task. The major limitations in using the existing approach are as follows:

\section{LIMITATIONS}

- When compared with the existing work, the accuracy values of the classifier are low.

- $\quad$ To predict the pedestrian based on the appearance is complicated due to large variation of appearance, poses and background clutter.

- Inaccurate prediction of pedestrian.
III

\section{PROPOSED METHODOLOGY}

In proposed methodology we make use of image threshold for characterising the gender of a walker.For training the proposed coordinate or system, all pictures that are present in the dataset are firstly preprocessed with person on foot parsing system.This method expels the unnecessary background information and creates the closer view cover that hold the passerby's full body.The closer view walker objects are gained by applying the front mask.These objects are useful for gender identifications of a pedestrian.

Random forest algorithm is used in this paper for identifying the pedestrian as a male or female we use this algorithm for classification cause .There are total number of 890 images in the MIT dataset that consists both male and female pictures having different views and they are front and back views. The pictures are of jpg pattern. The algorithm that is used in the paper predicts the pedestrian gender correctly.

Random Forest is a supervised learning algorithm which is utilized for both grouping and also for regression.So also,this algorithm makes choice trees on information tests and afterward gets the expectation from every one of them lastly chooses the best key by the process of voting.

The key computation for irregular random decision trees was made by Tin KamHo using the discretionary subspace technique that was in Ho's definition, is a way to deal with realize the "stochastic isolation" approach to manage request proposed by Eugene Kleinberg.

There are few advantages of this algorithm. They are

\section{ADVANTAGES}

- This algorithm is outstanding in accuracy among present algorithms.

- It runs adequately on enormous data bases.

- This can manage an enormous number of data factors without variable crossing out.

- This provides assessments of what components are noteworthy in the request.

- It has an effective procedure for assessing missing data and considers precision when a enormous degree of the data is missing.

A block diagram that is given below has a detailed explanation.In the next areas, a point by point conversation of all means of proposed system is introduced. 


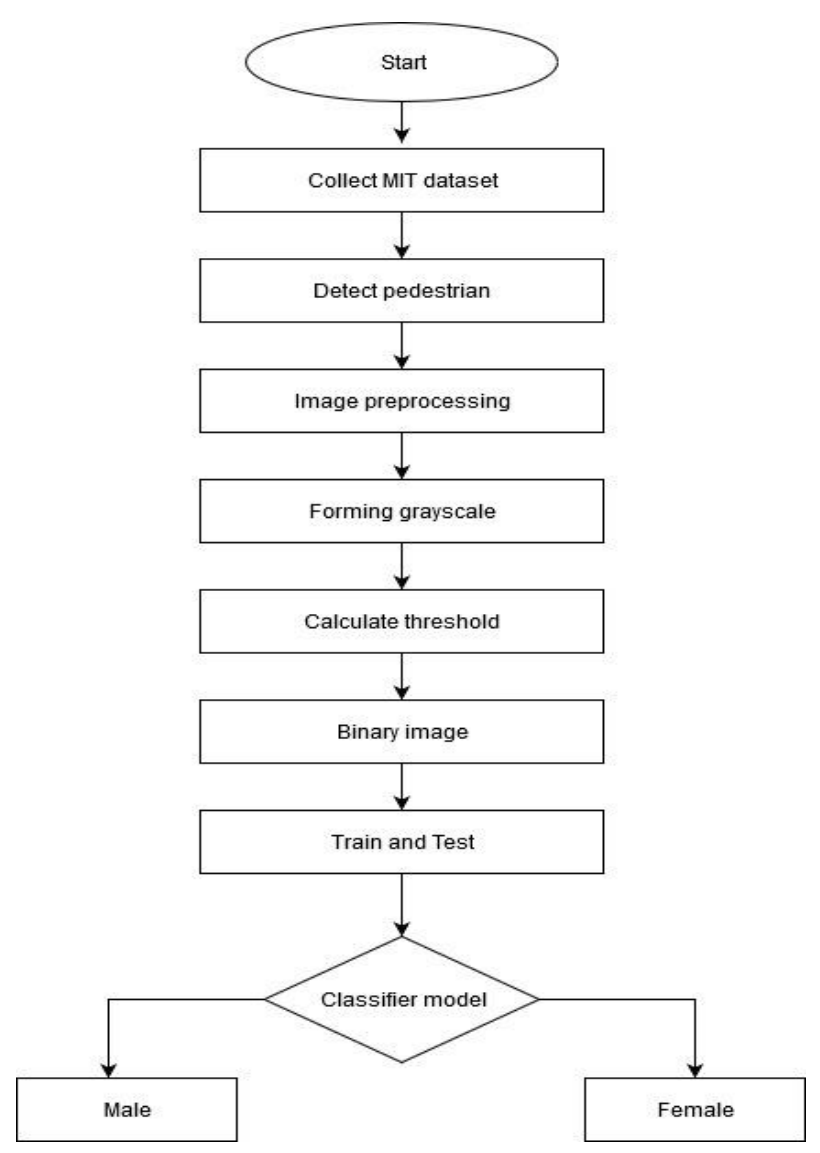

Figure 1: Dataflow diagram of the proposed methodology

MIT dataset is used in this paper for gender prediction of pedestrian. The above diagram gives the detailed explanation of the proposed methodology that has been used in this paper.

Firstly the MIT dataset is loaded in to spyder which contains 890 pictures with both male and female with front and back views. The images are in the format of jpg. Later a picture is displayed on running the program and grey scale conversion is done here in order to erase the background information so that it can appear clearly. Next outlier of a picture is obtained after grayscale image by calculating the threshold value for a image.

Later training and testing is performed on data and by using random forest classifier the gender of a walker or a pedestrian is classified as either men or women. This algorithm is systematic in terms of accuracy than other previous works.

\section{RESULTS}

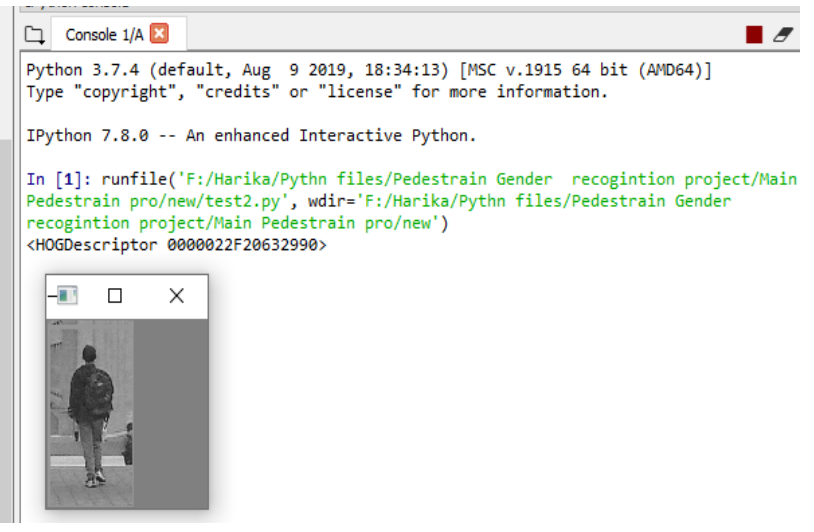

Figure : Greyscale image of a pedestrian

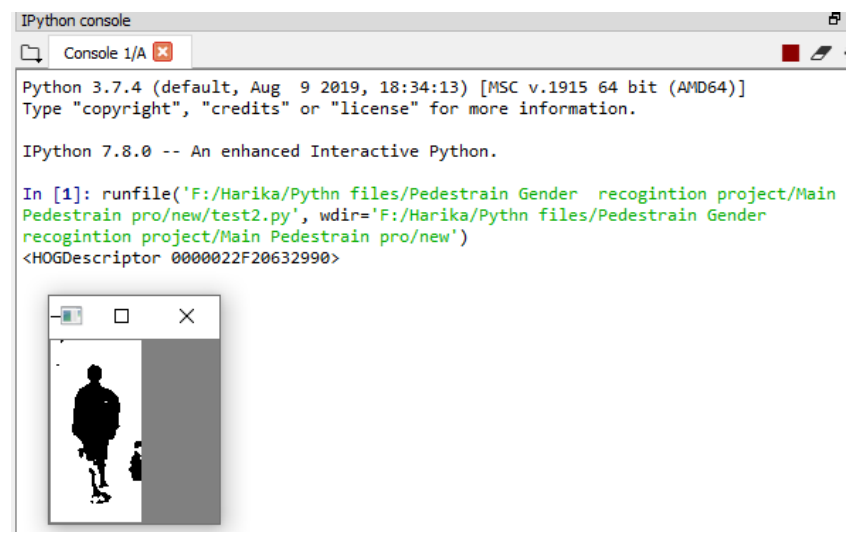

Figure : Binary image

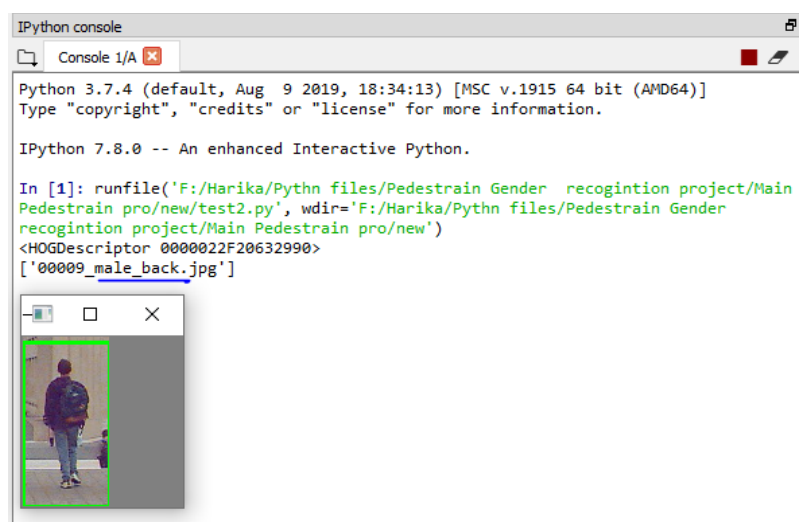

Figure: Prediction of gender output 


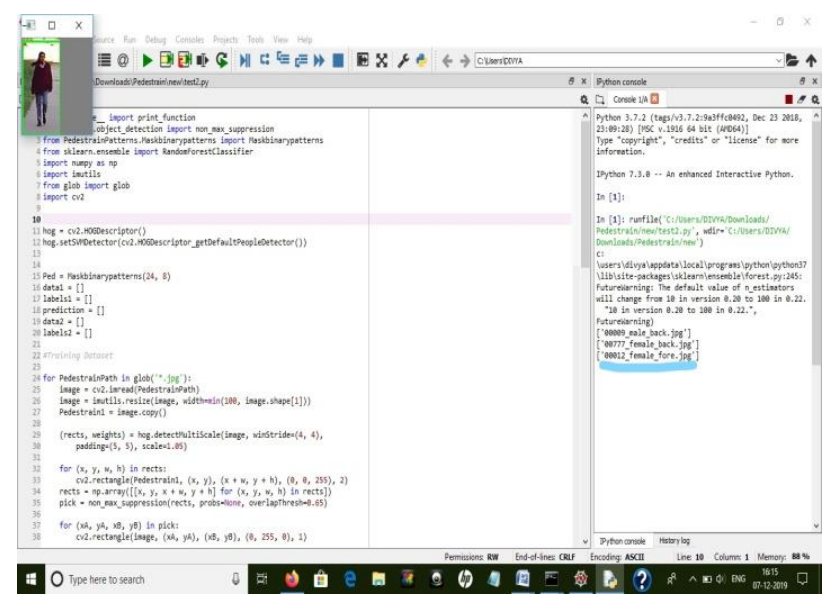

Figure: An Example of Gender Prediction

\begin{tabular}{|l|l|l|}
\hline Dataset & Algorithm & Accuracy \\
\hline MIT & Random Forest & $97.5 \%$ \\
\hline MIT & K -nearest neighbour & $20 \%$ \\
\hline
\end{tabular}

Table : Performance comparision

VI

\section{CONCLUSION}

In this project we focused on identifying the gender of a pedestrian as male or female by using appearance that uses the whole body. By applying the mask to the original input image the image of pedestrian is acquired and then background information of a image is erased by image preprocessing techniques. Later the acquired pictures are trained and tested on MIT dataset and by using the random forest classifier gender of a pedestrian is recognised. Previously many authors have provided their ideas for classifying the person but their research work is limited. In this research work we have used the random forest algorithm which gives 98 percent of accuracy in classification which is greater when compared with the other research works in this area. Inspite of the fact that the outcomes through proposed approach are improved, still this zone requires an immense research for additional upgrades.

\section{REFERENCES}

[1] S.A. Khan, M. Ahmad, M. Nazir, N. Riaz, A comparative analysis of gender classification techniques, Middle East J.Sci.Res.20(2014)113.

[2] Z. Shabbir, A.U. Khan, A. Irtaza, M.T. Mahmood, A fuzzy logic approach for gender recognition from face images with embedded bandlets, in: Artificial Intelligence and Soft Computing, Springer,2015, pp.626-637.

[3] R. Safdari, M.-S. Moin, A hierarchical feature learning for isolated farsi handwritten digit recognition using sparse auto encoder, in: Artificial Intelligence and Robotics (IRANOPEN),2016 ,IEEE,2016,pp.67-71.

[4] A.Ng, Sparse Auto Encoder, in : CS294 A Lecture notes, vol.72, 2011, pp.1-19.

[5] Y.Ju, J.Guo, S.Liu, A deep learning method combined sparse auto encoder with SVM, in: Cyber-Enabled Distributed Computing and Knowledge Discovery (CyberC), 2015 International Conference on, IEEE,2015,pp.257-260.

[6] C.I. Orozco, F. Iglesias, M.E. Buemi, J.J. Berlles, Real-Time Gender Recognition From Face Images Using Deep Convolutional Neural Network,2017.
[7] M.-C. Yang, C.-P. Wei, Y.-R. Yeh, Y.-C.F. Wang, Recognition at a long distance: Very low resolution face recognition and hallucination, in: Biometrics(ICB), 2015 International Conference on, IEEE, 2015,pp.237-242.

[8] W.W.Zou,P.C.Yuen,Very low resolution face recognition problem,IEETrans. ImageProcess.21(2012)327-340.

[9] G.Guo,G.Mu,Y.Fu,Gender from body:Abiologicallyinspired approach with manifold learning ,in: Computer Vision-ACCV 2009, Springer,2009,pp.236- 245.

[10] Fusion of probabilistic neuralnetwork and dictionary matching,Curr. Med. Imaging Rev.13(2017)176-184.

[11] S.L. Fernandes, G.J. Bala, Recognize faces across multi-view videos and under varying illumination, facial expressions, Int. J. Electron. Circuits Syst. Signal Pro.USA10(2016)7-18.

[12] S.L.Fernandes,J.G.Bala,Recognizing faces across age progressions and under occlusion,RecentPat.Comput.Sci.9(2016)209-215.

[13] S. Lawrence Fernandes, G. Josemin Bala, 3D and 4D face recognition: a comprehensive review, RecentPat.Eng.8(2014)112-119.

[14] S.L.Fernandes, G.J.Bala,Recognizing faces in corrupted images, in:Proce edings of the International Conference on Soft Computing Systems, Springer, 2016, pp.511-518.

[15] R. Ranjan, V.M. Patel, R. Chellappa,Hyperface: A deep multi-task learning framework for face detection, landmark localization, pose estimation, and gender recognition, IEEE Trans.Pattern Anal. Mach.Intell.(2017). 